\title{
Tracheostomy protocols during COVID-19 outbreak
}

\author{
Cameron P. Heyd et al. ${ }^{1}$ \\ ${ }^{1}$ Affiliation not available
}

April 28, 2020

\begin{abstract}
Background: The novel coronavirus, severe acute respiratory syndrome coronavirus 2 (SARS-Cov-2) is spread through aerosol and fine droplets, and poses many challenges to medical practitioners. Otolaryngologists are at an exceptionally high-risk, due to common aerosol-generating procedures such as tracheostomy. The purpose of this study was to evaluate clinical guidelines for tracheostomy in reference to SARS-CoV-2 and provide a collective summary of recommendations.
\end{abstract}

Methods: Literature review was performed. Articles reporting clinical practice guidelines for tracheostomy in the context of SARS-CoV-2 were included.

Results: Tracheostomies are a common surgical procedure performed by otolaryngologists. There may be expanding indications in the COVID-19 patient population. Ventilation using a tracheostomy has many potential benefits and a summary of recommendations for tracheostomy (elective or emergent) and tracheostomy management in COVID-19 positive patients are detailed within this article.

Conclusions: In patients testing positive for COVID-19, this summary of recommendations serves as a guideline along with institutional protocols.

\section{Authors and affiliations}

\section{Introduction}

The first cases of severe acute respiratory syndrome coronavirus 2 (SARS-CoV-2), the virus causing coronavirus disease (COVID-19), began as only a few cases in rural China and has now grown into a global pandemic. While this virus does not appear to be as deadly as the coronavirus outbreak in 2003 known as SARS, it unfortunately has proven to be much more infectious. SARS-CoV-2 has an incubation period of an estimated 4 days and a relatively slow onset of symptoms, allowing infected persons to unknowingly transmit the virus (1).

Although most cases range from relatively asymptomatic to mild flu-like symptoms, approximately $20-30 \%$ of COVID-19 patients require admission to the intensive care unit (ICU) for respiratory support (2). This rapid influx of patients has challenged institutions and medical practitioners alike. In response, many guidelines continue to be updated by the Centers for Disease Control and Prevention (CDC), World Health Organization (WHO), and individual societies from around the world.

Due to the spread of SARS-CoV-2 through aerosol and fine droplets, medical personnel are in direct danger of occupational exposure while caring for these patients. This is especially true for aerosol-generating airway procedures which can potentially expose everyone in the room (3). A report from the outbreak in Wuhan, China warns that otolaryngologists are exceptionally at risk, citing an event in which 14 medical personnel contracted COVID-19 during an endoscopic pituitary surgery (4). Therefore, the risk posed to otolaryngologists during many commonly performed surgeries cannot be understated. A statement from American Academy of Otolaryngology - Head and Neck Surgery "strongly recommends that all otolaryngologists provide only time-sensitive or emergent care" in order to mitigate this risk (5). Tracheostomies 
and tracheostomy care, however, play a critical role in the management of COVID-19 patients: electively to provide closed-circuit ventilation in prolonged endotracheal intubation or emergently for airway access. These interventions are necessary to provide adequate care, but they also demand special precautions be taken in order to mitigate occupational risk.

The purpose of this study was to evaluate the current practice guidelines and recommendations in regards to SARS-CoV-2 as they pertain to tracheostomy and provide a collective summary of recommendations. Individual guidelines have been published from groups around the world to guide medical personnel during aerosol-generating procedures, such as a tracheostomy. It is essential that all those potentially involved are aware of these guidelines and implement them when appropriate.

\section{Methods}

\section{Literature Review and Evidence Collection}

A literature review was performed by one author, searching for all published English-language literature reporting on clinical practice guidelines pertaining to tracheostomy in the context of COVID-19. A search strategy was employed with the following search strings: "covid OR covid-19 OR coronavirus" with "tracheostomy OR tracheotomy OR tracheostomy change" in the last 5 years. Grey literature (literature that is not commercially published) was identified by searching the websites of governmental health agencies, professional associations, and other medical societies. Google search engine was used to search for additional information. These searches were supplemented by references of relevant individual articles. Results were limited to articles in the English language. After the completed search, a total list of records was obtained, and duplicates removed. A final list of full text articles was then compiled, and one author independently screened each article.

\section{Discussion}

Tracheostomies are a common surgical procedure performed by otolaryngologists in both the emergent and elective setting. Additionally, there may be some expanding indications in the COVID-19 patient population. As COVID-19 patients wean off the ventilator, some may be at high risk for requiring non-invasive ventilation or reintubation. Both instances have been noted to be aerosol-generating events, and should be avoided when possible. Ventilation using a tracheostomy has many potential benefits including providing closed-circuit respiratory support, allowing for decreased sedation, and requiring less intensive nursing care (6). Discussion between intensive care and otolaryngology teams should take place to decide whether tracheostomy is indicated in this patient population. In patients testing positive for COVID-19, this summary of recommendations serves as a guideline along with institutional protocols.

\section{Elective Tracheostomy in COVID-19 positive patients}

\section{GENERAL PRECAUTIONS}

- Allow adequate time for education and rehearsal with staff prior to donning necessary PPE, as N95 masks can make communication difficult. This will ensure all involved parties are adequately informed and operate smoothly (7-14).

- Tracheostomy should be avoided in patients with respiratory instability or heavy ventilator dependence (6).

- If resources allow, test all tracheostomy patients for COVID-19 within 48 hours prior to surgery (7, 8, $10,12-14)$.

- COVID-19 positive patients should postpone elective cases until testing negative, if possible (7-16).

- Airborne precautions should be followed by all team members that are present (7-16).

\section{LOCATION}

- The operation should be performed in an ICU room or operating room, preferably with negative pressure and a HEPA filtration system. If performed in an ICU room, be aware the width of an 
ICU bed might limit surgical access. Consider positioning the patient closer to the surgeon prior to beginning the procedure $(7,10,11,13-16)$.

- If necessary to move the patient, care should be taken when transporting the patient to the operating room. Designate a team member to remain clear of contact with the patient and interact with the environment.

\section{PERSONNEL}

- Reduce team members to only essential staff. Consider one surgeon, one anesthesiologist, and one surgical staff member. Additional team members may remain on standby outside of the room (7-13).

- Procedure should be performed by the most experienced staff to maximize safety and efficiency. Trainees only to be present if no attendings available $(7-12,16)$.

- Consider forming a "traveling trach team" to limit inexperienced staff during COVID-19 positive procedures $(7,10,13)$.

\section{EQUIPMENT}

- All personnel should be equipped with the proper PPE, including an N95 mask or purified air purifying respirator (PAPR) device, eye goggles, transparent full-face shield, gown, and double gloves (7-16).

- PPE equipment should be readily available in the area where COVID-19 positive patients are managed (7-16).

- Surgical tracheotomy tray (17).

- Cuffed tracheostomy set (7-12).

- Heat and moisture exchanger (HME) device with viral filter (7-16).

- Closed suction system $(7,8,10-16)$.

\section{INTRAOPERATIVE TECHNIQUES}

- Limit use of electrical cautery (17).

- Maintain adequate preoxygenation $(100 \%)$ for 5 minutes $(8-11,13,16)$.

- Establish complete paralysis using neuromuscular blockade to prevent coughing and aerosol dispersion $(3,5,6,10,13,14)$.

- Hold ventilation prior to puncturing airway $(7,12)$.

- Once tracheostomy placement is confirmed, connect patient to ventilator with HME/viral filter (8-11, 13-16).

- Suture the tube to the skin and strap separately (17).

- After removing endotracheal tube, place facemask on patient to protect against dispersion should the patient cough (14).

- Check for proper inflation and monitor any evidence of cuff leaks $(6,7,12,15)$.

- Doffing of PPE and disposal of tools according to institutional protocol (6-17).

\section{Emergency Tracheostomy in COVID-19 positive or unknown patient}

In the event of an airway emergency in which endotracheal intubation is unable to be achieved and a tracheostomy is indicated, the guidelines above should be followed. An emphasis should be placed on safety of medical personnel through the proper use of PPE. Reviewal and implementation of these guidelines can limit confusion and unnecessary occupational exposure to staff $(7,12,17)$.

\section{Tracheostomy management in COVID-19 positive patients}

\section{GENERAL PRECAUTIONS}

- Patients should be managed as patients with COVID-19 who require oxygenation/hospitalization (7).

- Encourage the patient to perform as much of the cleaning and care as they are comfortable (7).

- Staff caring for this patient set should be properly educated on COVID-19 airway management (such as using closed suctioning, cannula care), as these are high-risk (to staff) scenario $(7,12)$.

- PPE and airborne/droplet precautions should be followed according to institutional protocol (6-17). 
- Tracheostomy change procedures should be delayed until patient no longer tests positive, if possible $(6,7,12)$.

\section{VENTILATED PATIENTS}

- Avoid disconnecting patient circuit from ventilator. If necessary, clamp tubing distal to HME filter prior to disconnecting $(8-12,14,15)$.

- Use inner cannula if thick secretions or on open system. If used, limit inspection/cleaning of inner cannula (7).

- Cuff deflation when weaning from ventilator will result in aerosol generation. Ensure patients are in isolation or in a cohort room with other COVID-19 patients (7).

\section{NON-VENTILATED PATIENTS}

- Cuffed, non-fenestrated tracheostomy tubes should be used and remain inflated to limit aerosol generation $(7-9,11,12,17)$.

- Humidification with a simple HME device, along with a viral filter should be placed on the tracheostomy tube (6-17).

- A simple facemask should be placed on the patient $(7,13-15)$.

- Any suctioning should be done using a closed system (7-12, 14-17).

- Should supplemental oxygenation be needed, a trach collar is preferred due to its protection against droplet spread (7).

\section{Limitations}

This study evaluated the current practice guidelines and recommendations for tracheostomy during the SARS-CoV-2 outbreak to provide a collective summary for otolaryngologists in this difficult time. However, we acknowledge the inherent limitations of this study. Although we included all published literature to date, this was limited only to those published before March 31st, 2020. As more is learned about SARS-CoV-2 and more data becomes available, it is possible that the guidelines may change or be altered as a result. Still, this is the most up-to-date collection of clinical guidelines for tracheostomy with respect to the SARS-CoV-2 outbreak available at this time. Further studies are needed to determine the efficacy of these guidelines, and in order to establish recommendations for similar viral epidemics in the future.

\section{References}

1. Guan WJ, Ni ZY, Hu Y, Liang WH, Ou CQ, He JX, et al. Clinical Characteristics of Coronavirus Disease 2019 in China. The New England journal of medicine. 2020.

2. Yang X, Yu Y, Xu J, Shu H, Xia Ja, Liu H, et al. Clinical course and outcomes of critically ill patients with SARS-CoV-2 pneumonia in Wuhan, China: a single-centered, retrospective, observational study. The Lancet Respiratory Medicine. 2020.

3. Tran K, Cimon K, Severn M, Pessoa-Silva CL, Conly J. Aerosol generating procedures and risk of transmission of acute respiratory infections to healthcare workers: a systematic review. PloS one. 2012;7(4):e35797.

4. Zara M. Patel MJF-M, MD; Peter H. Hwang, MD; Jayakar V. Nayak, MD, PhD; Robert Dodd, MD, PhD; Hamed Sajjadi, MD; Robert K. Jackler, MD. Precautions for endoscopic transnasal skull base surgery during the COVID-19 pandemic. 2020.

5. Otolaryngologists and the COVID-19 Pandemic. American Academy of Otolaryngology - Head and Neck Surgery. 2020.

6. Noah P. Parker MBAS, MD; Mark A. Fritz, MD; Sarah K. Rapoport, MD; Sam Schild, MD; Kenneth W. Altman, MD; Albert L. Merati, MD; Maggie A. Kuhn, MD. Tracheotomy recommendations during the COVID-19 Pandemic. American Academy of Otolaryngology - Head and Neck Surgery. 2020. 
7. NTSP considerations for tracheostomy in the Covid-19 outbreak. National Tracheostomy Safety Project. 2020 .

8. COVID-19 Resources for Anesthesiologists. American Society of Anesthesiologists. 2020.

9. Anaesthesiologists WFoSo. Coronavirus - guidance for anaesthesia and perioperative care providers 2020.

10. David J Brewster NCC, Thy BT Do, Kirstin Fraser, Chris J Groombridge, Andy Higgs, Matthew J Humar, Timothy J Leeuwenburg, Steven McGloughlin, Fiona G Newman, Chris P Nickson, Adam Rehak, David Vokes and Jonathan J Gatward. Consensus statement: Safe Airway Society principles of airway management and tracheal intubation specific to the COVID-19 adult patient group. The Medical Journal of Australia. 2020.

11. Kong DoAaIC-TCUoH. Infographic for Principles of Airway Management in COVID-19. 2020.

12. Laura Harrison JR, Stuart Winter. Guidance for surgical tracheostomy and tracheostomy tube change during the COVID-19 Pandemic. ENT UK. 2020.

13. Wax RS, Christian MD. Practical recommendations for critical care and anesthesiology teams caring for novel coronavirus (2019-nCoV) patients. Canadian Journal of Anesthesia/Journal canadien d'anesthésie. 2020 .

14. Xiangdong Chen MD, Ph.D.; Yanhong Liu, M.D., Ph.D.; Yahong Gong, M.D.; Xiangyang Guo, M.D., Ph.D.; Mingzhang Zuo, M.D., Ph.D.; et al. Perioperative Management of Patients Infected with the Novel Coronavirus: Recommendation from the Joint Task Force of the Chinese Society of Anesthesiology and the Chinese Association of Anesthesiologists. Anesthesiology. 2020.

15. [Expert consensus on preventing nosocomial transmission during respiratory care for critically ill patients infected by 2019 novel coronavirus pneumonia]. Zhonghua jie he he hu xi za zhi = Zhonghua jiehe he huxi zazhi $=$ Chinese journal of tuberculosis and respiratory diseases. 2020;17(0):E020.

16. Liana Zucco NL, Desire Ketchandji, Mike Aziz, Satya Krishna Ramachandran. Perioperative Considerations for the 2019 Novel Coronavirus (COVID-19) Anesthesiology Patient Safety Foundation. 2020.

17. Wei WI, Tuen HH, Ng RWM, Lam LK. Safe tracheostomy for patients with severe acute respiratory syndrome. The Laryngoscope. 2003;113(10):1777-9. 